\title{
$P_{2 k}-$ Factorization Induced Network Flow
}

\author{
U.S. Rajput \\ Department of mathematics and astronomy, \\ Lucknow University, Lucknow (India).
}

\begin{abstract}
$P_{k}$-factorizations of complete bipartite graph have been studied by several researchers. For even value of $k$, the spectrum problem is completely solved [6,7]. Here in this paper we will obtain a feasible network flow of $P_{2 k}$-factorization of a complete bipartite graph satisfying the conditions of $P_{2 k}$-factorization. In this paper we construct the disjoint flow paths in $P_{2 k}$-factorization of complete bipartite graph $K_{m, n}$ (for $k=1$ and 2). We deduce that $P_{2 k}$-factorization of complete bipartite graph is helpful in finding the disjoint flow paths in a compete bipartite graph $K_{m, n}(m=n)$. The result can be generalized for any value of $k$ with $m=n$.
\end{abstract}

\section{Mathematics Subject Classification 2010: 68R10, 05C21, 05C70.}

\section{Keywords}

Complete bipartite Graph, Factorization of Graph, Network Flow

\section{INTRODUCTION}

Let $K_{m, n}$ be the complete bipartite graph with two partite sets having $m$ and $n$ vertices, respectively. A spanning subgraph $F$ of $K_{m, n}$ is called a path factor if each component of $F$ is a path of order at least two. For any positive integer $k$, a path on $2 k$ vertices is denoted by $P_{2 k}$. In particular a spanning sub graph $F$ of $K_{m, n}$ is called a $P_{2 k}$-factor of $K_{m, n}$ if each component of $F$ is isomorphic to $P_{2 k}$. If $K_{m, n}$ is expressed as an arc disjoint sum of $P_{2 k}$-factors, then this sum is called a $P_{2 k}$-factorization of $K_{m, n}$.

A Network flow is called feasible flow with source $s$ and sink $t$ if it satisfies the following two condition as given by $[3,4]$.

$$
\begin{aligned}
& \text { (i) } 0 \leq f(u, v) \leq c(u, v) \quad \forall(u, v) \in E \text {. These are } \\
& \text { the capacity constraints. (If a capacity is } \infty \text {, } \\
& \text { then there is no upper bound on the flow value } \\
& \text { on that edge.) } \\
& \text { (ii) For all } v \in V-\{s, t\} \text {, the total flow into } v \text { is }
\end{aligned}
$$
same as the total flow out of $v$ :

$$
\sum_{u:(u, v) \in E} f(u, v)=\sum_{w:(v, w) \in E} f(v, w) .
$$

Constraint ( $i i$ ) is called flow conservation law for network flow graph. The conservation law holds at all vertices other than the source and the sink. The value of flow denoted by $\operatorname{val}(f)$, is the net flow out of the source:

\author{
Bal Govind Shukla
nt of mathematics and astronomy,
w University, Lucknow (India). \\ Bal Govind Shukla
Department of mathematics and astronomy,
Lucknow University, Lucknow (India). \\ Bal Govind Shukla
Lucknow University, Lucknow (India).
}

$$
\operatorname{val}(f)=\sum_{u:(s, u) \in E} f(s, u)
$$

In $P_{2 k}$-factorization induced network flow, each path factor of complete bipartite graph $K_{m, n}$ will contribute to a flow path, and hence this network flow will be a collection of flow paths, each of which is an undirected path factor. We assume capacity of each edge as one which is not shown in the figures drawn.

\section{MATHEMATICAL ANALYSIS}

In the study of $P_{2 k}-$ path factorization of complete bipartite graph $K_{m, n}$, we find different mutually vertex/edge disjoint paths. Each path will be a flow path. For each value of $k$ (even/odd) flow path can be developed. In this paper we consider particular cases of even and odd values of $k$. For simplicity, we are considering only $P_{2}$ and $P_{4}$ factorization.

Network Flow in $\boldsymbol{P}_{2 \boldsymbol{k}}$-factorization: Let $P_{2 k}$ be a path on even vertices and $K_{m, n}$ be a complete bipartite graph with partite sets having $m$ and $n$ vertices. Wang [9] gave the necessary and sufficient conditions for the existence of a $P_{2 k}$-factorization of $K_{m, n}$ when $m=n$. Which are given in theorem 2.1 below.

Theorem 2.1: $K_{m, n}$ has $P_{2 k}$-factorization if and only if $m=n$ and $m \equiv 0(\bmod k(2 k-1))$.

To show the feasible network flow in $P_{2 k}$-factorization of $K_{m, n}$, we consider $k=1, m=4$ and $n=4$, Fig. 1 .

Here let $t$ be the number of copies in a $P_{2}$-factor graph, then in this case

$$
\begin{gathered}
t=\frac{m+n}{2} \\
=\frac{4+4}{2}=4
\end{gathered}
$$

And $r$ be the number of disjoint $P_{2}$-factors in graph, i.e.

$$
\begin{aligned}
& r=\frac{2 m n}{m+n} \\
& =\frac{2 \times 4 \times 4}{8}=4 .
\end{aligned}
$$

To make it disjoint network flow graph we add source and sink in $P_{2}$-factorization of $K_{4,4}$. 


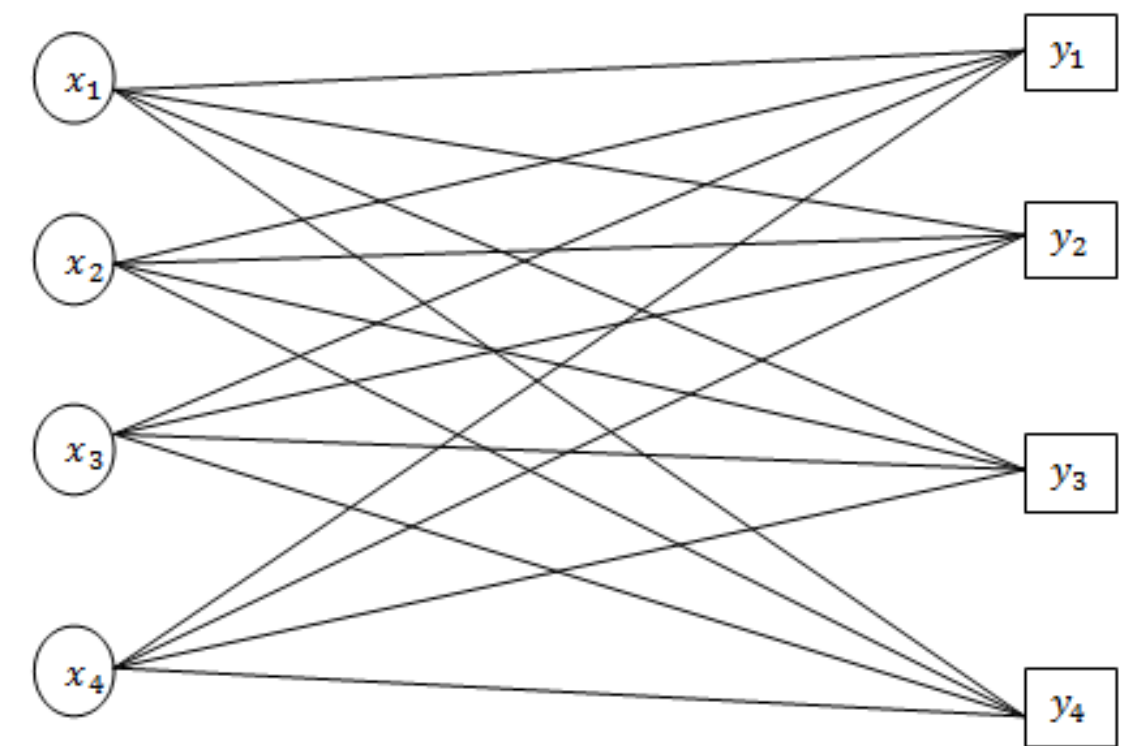

Fig. 1: Complete bipartite graph $K_{4,4}$

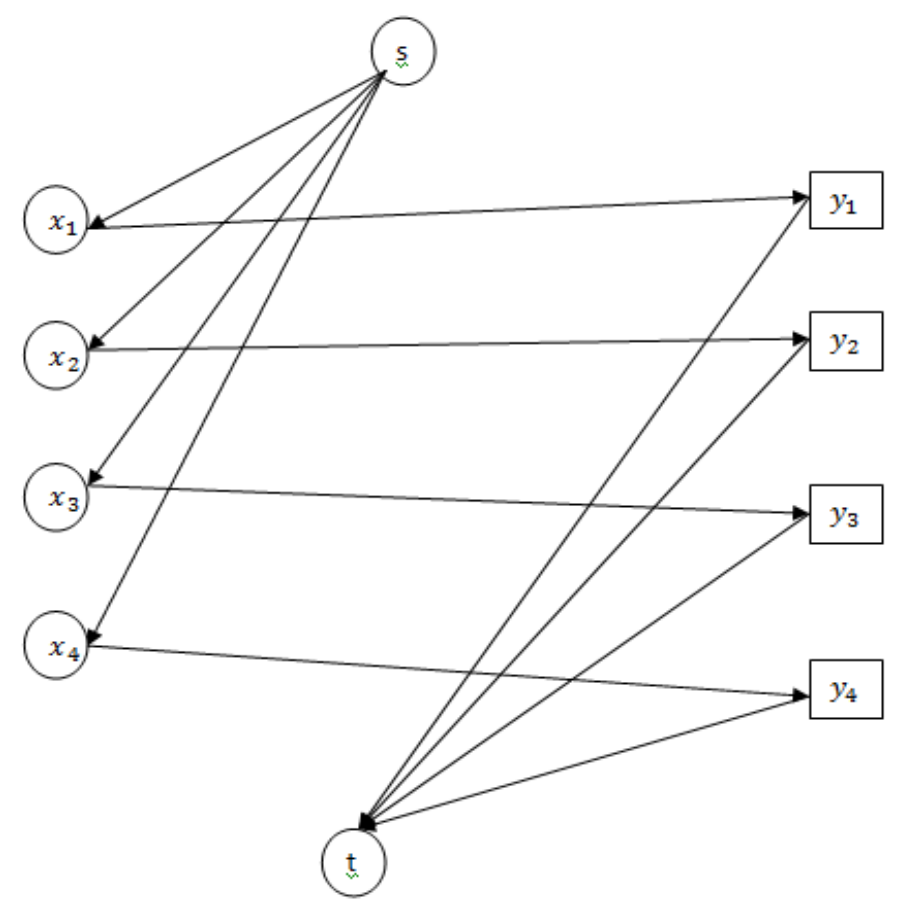

Fig. 2: Flow path $s x_{1} y_{1} t, s x_{2} y_{2} t, s x_{3} y_{3} t, s x_{4} y_{4} t$. 


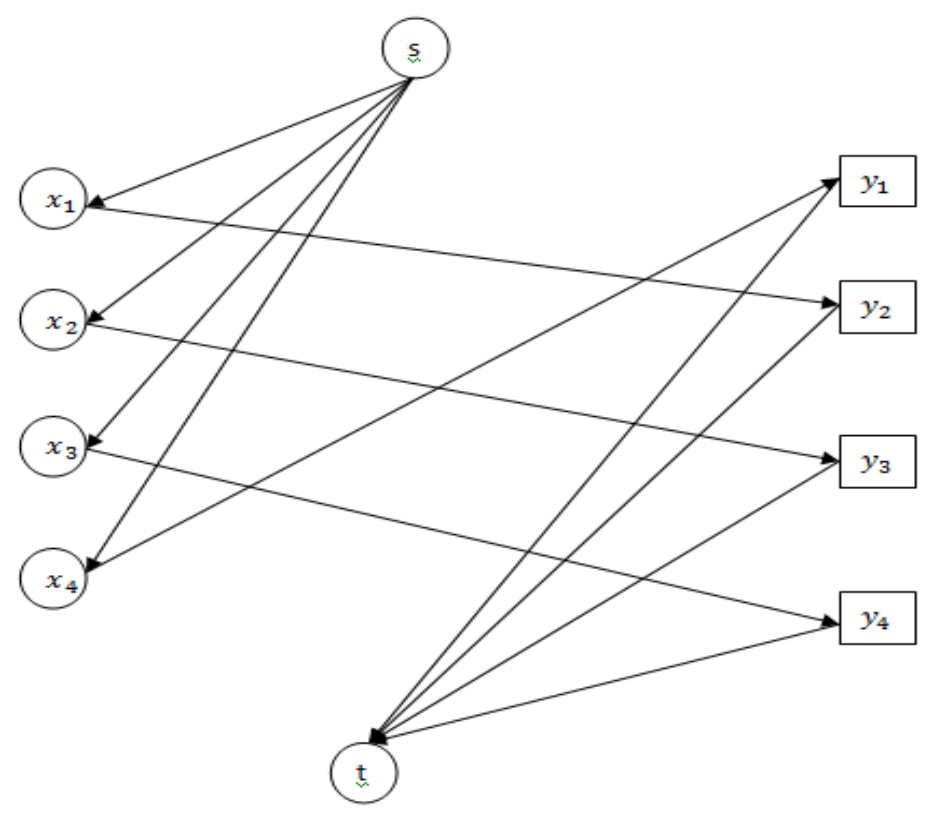

Fig. 3: Flow path $s x_{1} y_{2} t, s x_{2} y_{3} t, s x_{3} y_{4} t, s x_{4} y_{1} t$.

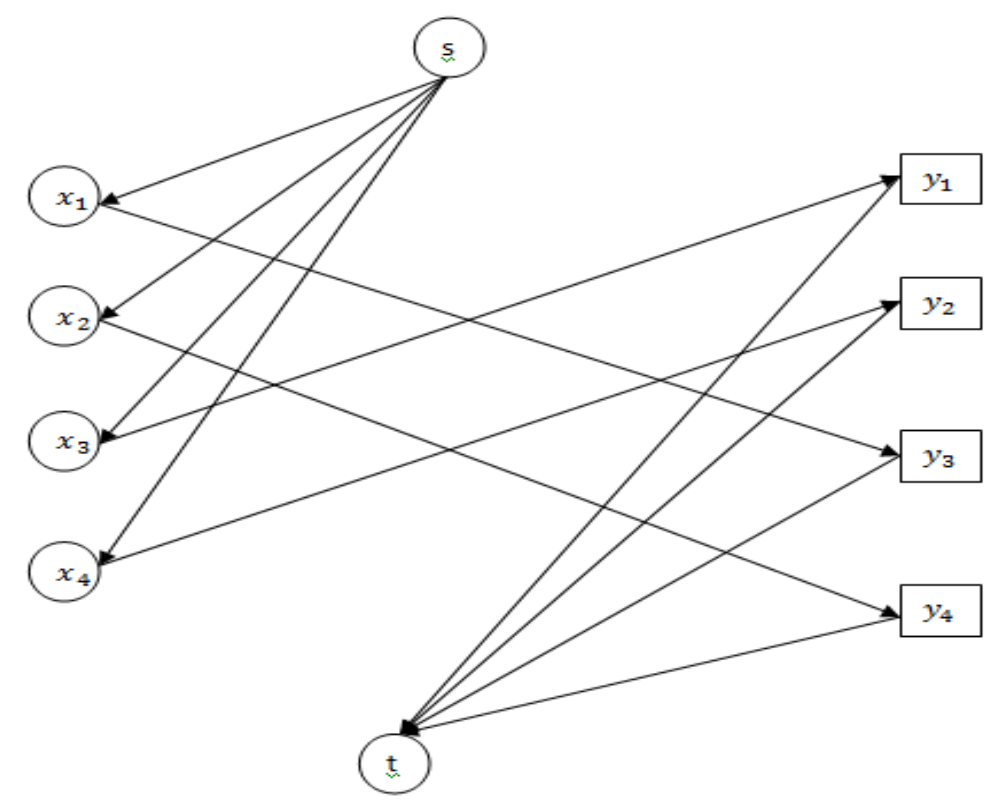

Fig. 4: Flow path $s x_{1} y_{3} t, s x_{2} y_{4} t, s x_{3} y_{1} t, s x_{4} y_{2} t$. 


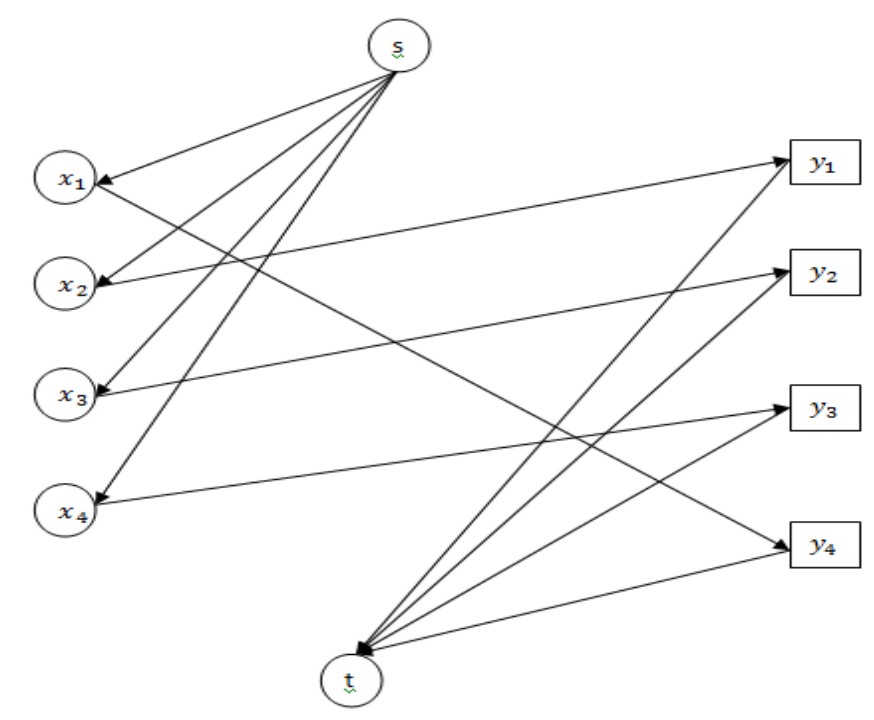

Fig. 5: Flow path $s x_{1} y_{4} t, s x_{2} y_{1} t, s x_{3} y_{2} t, s x_{4} y_{3} t$.

As shown above in Fig. 1 to Fig. 5, we find the disjoint feasible network flow path between source $\mathrm{s}$ and sink $\mathrm{t}$ in $P_{2}$-factorization of $K_{2,2}$.

Similarly, for $P_{4}$-factorization of $K_{m, n}$, we consider $k=$ $2, m=6$ and $n=6$ (Theorem -2.1), Fig. 4 .

Here in this case let $t$ be the number of copies in a $P_{4}-$ factor graph, then

$$
t=\frac{m+n}{4}
$$

$$
=\frac{6+6}{4}=3
$$

and $r$ be the number of disjoint $P_{4}$-factors in graph, i.e.

$$
\begin{aligned}
r & =\frac{2 m n}{3(m+n)} \\
& =\frac{4 \times 6 \times 6}{3 \times 12}=4 .
\end{aligned}
$$

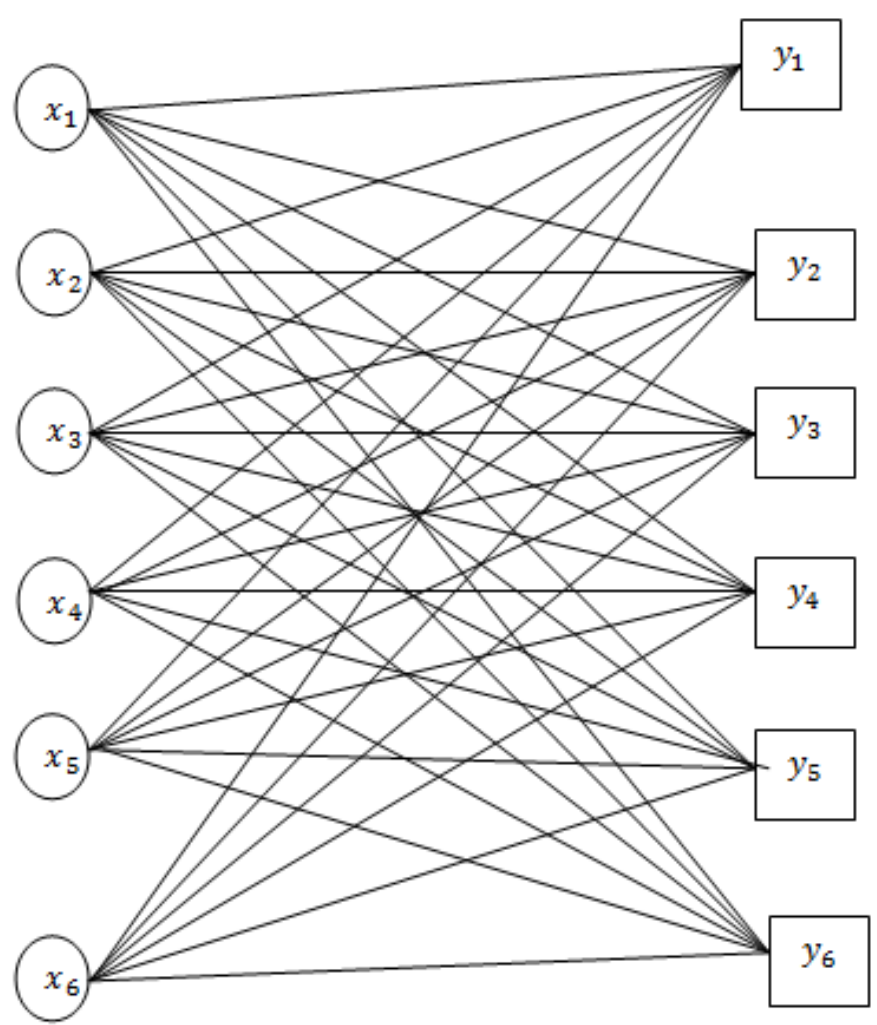

Fig. 6: Complete bipartite graph $K_{6,6}$ 


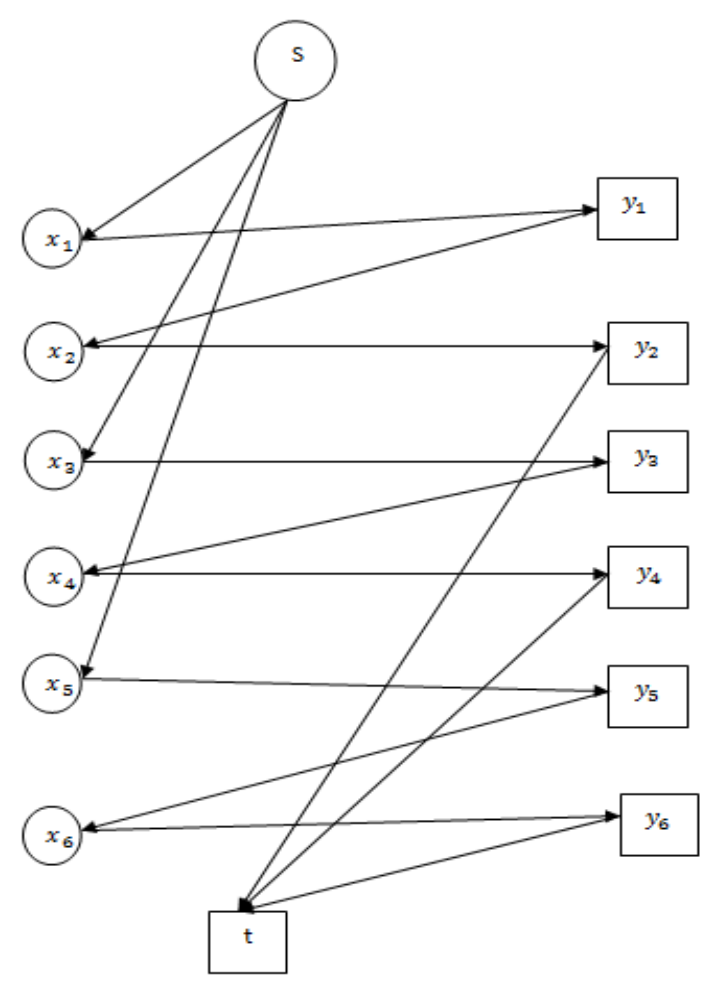

Fig. 7: Flow paths $s x_{1} y_{1} x_{2} y_{2} t, s x_{3} y_{3} x_{4} y_{4} t, s x_{5} y_{5} x_{6} y_{6} t$.

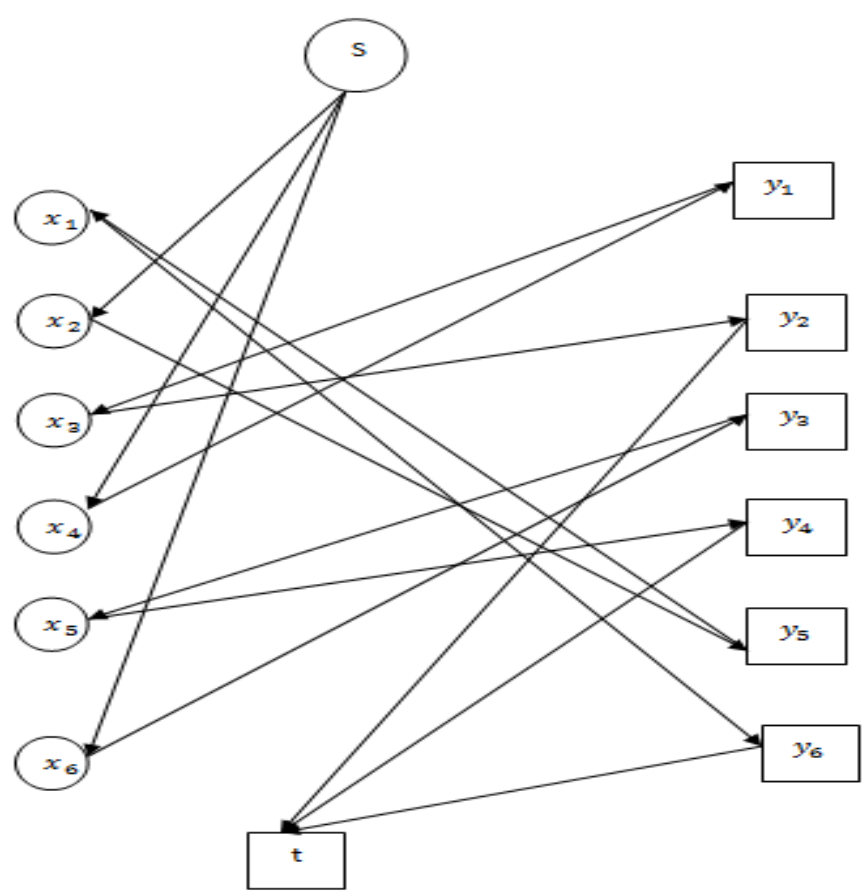

Fig. 8: Flow paths $s x_{4} y_{1} x_{3} y_{2} t, s x_{6} y_{3} x_{5} y_{4} t, s x_{2} y_{5} x_{1} y_{6} t$. 


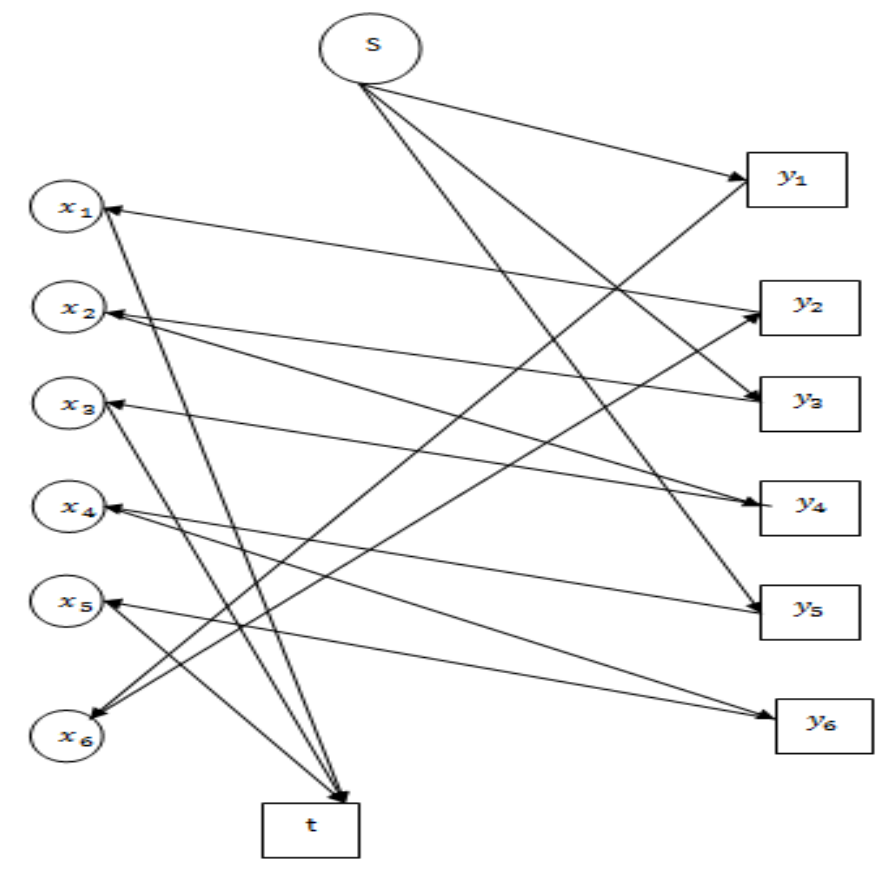

Fig. 9: Flow paths $s y_{1} x_{6} y_{2} x_{1} t, s y_{3} x_{2} y_{4} x_{3} t, s y_{5} x_{4} y_{6} x_{5} t$.

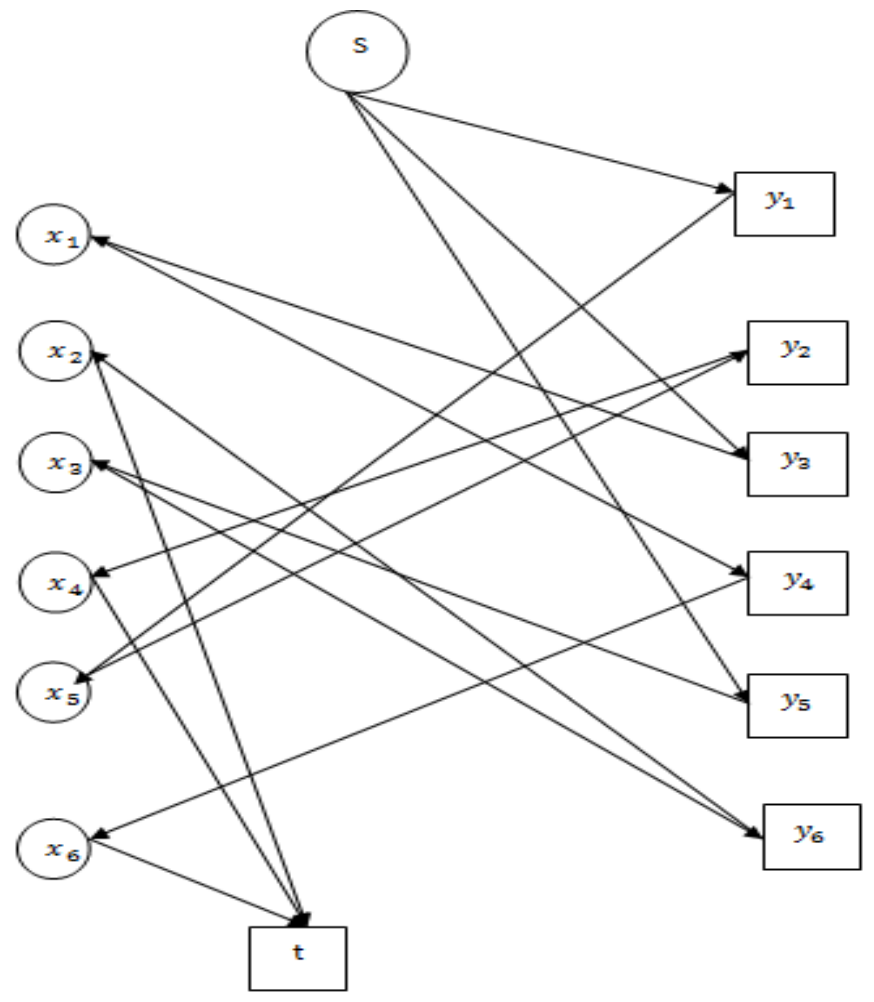

Fig. 10: Flow Paths $s y_{1} x_{5} y_{2} x_{4} t, s y_{3} x_{1} y_{4} x_{6} t, s y_{5} x_{3} y_{6} x_{2} t$. 


\section{DISCUSSION}

In this paper it is analyzed that $P_{2 k}$-factorization of complete bipartite graph $K_{m, n}$ (for $k=1$ and 2), will give the disjoint flow paths. Hence we deduce that $P_{2 k}$-factorization of complete bipartite graph is helpful in finding the disjoint flow paths in a complete bipartite graph $K_{m, n}$ when $m=n$. The result can be generalized for any value of $k$ with $m=n$.

\section{REFERENCES}

[1] Beliang Du: $P_{2 k}$-factorization of complete bipartite multigraph. Australasian Journal of Combinatorics 21(2000), 197 - 199.

[2] Du B and Wang J: $P_{4 k-1}$-factorization of complete bipartite graphs. Science in China Ser. A Mathematics 48 (2005) 539 - 547.

[3] Ford L R, Fulkerson D R. "Maximum Flow through a Network". Canadian journal of Mathematics 8:399(1956).

[4] Fulkerson D R ; "Flow Network and Combinatorial Operations Research". Amer. Math. Monthly 73. 115138(1966).

[5] Rajput U S and Shukla Bal Govind: $P_{9}$-factorization of complete bipartite graphs. Applied Mathematical
Sciences, volume 5(2011), 921- 928.

[6] Rajput U S and Shukla Bal Govind: $P_{4 k+1}-$ factorization of complete bipartite graphs: Elixir Dis. Math. 45 (2012) 7893-7897.

[7] Ushio K: $P_{3}$-factorization of complete bipartite graphs. Discrete math.72 (1988) 361-366.

[8] Ushio K: $G$-designs and related designs, Discrete Math., 116(1993), 299-311.

[9] Wang H: $P_{2 p}$-factorization of a complete bipartite graph, discrete math.120 (1993) 307-308.

[10] Wang $\mathrm{J}$ and $\mathrm{Du} \mathrm{B}$ : $P_{5}$-factorization of complete bipartite graphs. Discrete math. 308 (2008) $1665-1673$.

[11] Wang J: $P_{7}$-factorization of complete bipartite graphs. Australasian Journal of Combinatorics, volume 33 (2005), 129-137.

[12] Ushio K. Path-factorization of complete bipartite graphs, Discrete Math., 72(1988),361-366.

[13] Bondy J A and Murty U S R. Graph Theory with Applications, ( Macmillan, London and Basingstoke, 1976) $72-75$. 